\title{
Carcinogenic liver fluke Opisthorchis viverrini oxysterols detected by LC-MS/MS survey of soluble fraction parasite extract ${ }^{\text {}}$ s
}

\author{
Nuno Vale a,*, Maria João Gouveia ${ }^{\text {b }}$, Mónica Botelho b,c, Banchob Sripa d , Sutas Suttiprapa e, Gabriel Rinaldi f,g, \\ Paula Gomes ${ }^{\mathrm{a}}$, Paul J. Brindley ${ }^{\mathrm{f}}$, José Manuel Correia da Costa ${ }^{\mathrm{b}, \mathrm{c}}$ \\ a CIQUP, Chemistry and Biochemistry Department, Faculty of Sciences, University of Porto, Rua Campo Alegre, 687, 4169-007 Porto, Portugal \\ b Center for the Study of Animal Science, ICETA, University of Porto, Rua de Jorge Viterbo Ferreira, 228, 4050-313 Porto, Portugal \\ ' INSA, Rua Alexandre Herculano, 321, 4000-055 Porto, Portugal \\ d Department of Pathology, Faculty of Medicine, Khon Kaen University, Khon Kaen 40002, Thailand \\ e Department of Microbiology, Faculty of Science, Mahidol University, Bangkok 10400, Thailand

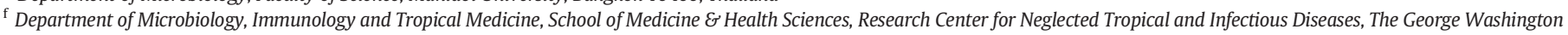 \\ University, Washington, DC 20037, USA \\ g Departamento de Genética, Facultad de Medicina, Universidad de la República (UDELAR), Montevideo 11800, Uruguay
}

\section{A R T I C L E I N F O}

\section{Article history:}

Received 4 December 2012

Received in revised form 2 August 2013

Accepted 7 August 2013

Available online 20 August 2013

\section{Keywords:}

Bile acids

Flukes

Opisthorchis viverrini

Oxysterols

\begin{abstract}
A B S T R A C T
Liquid chromatography in tandem mass spectrometry (LC-MS/MS) has emerged as an informative tool to investigate oxysterols (oxidized derivatives of cholesterol) in helminth parasite associated cancers. Here, we used LCMS/MS to investigate in soluble extracts of the adult developmental stage of Opisthorchis viverrini from experimentally infected hamsters. Using comparisons with known bile acids and the metabolites of estrogens, the LC-MS data indicated the existence of novel oxysterol derivatives in 0 . viverrini. Most of these derivatives were ramified at C-17, in similar fashion to bile acids and their conjugated salts. Several were compatible with the presence of an estrogen core, and/or hydroxylation of the steroid aromatic ring A, hydroxylation of both C-2 and C-3 of the steroid ring and further oxidation into an estradiol-2,3-quinone.
\end{abstract}

(c) 2013 The Authors. Published by Elsevier Ireland Ltd. All rights reserved.

\section{Introduction}

Liver fluke infection caused by Opisthorchis viverrini, Clonorchis sinensis and related flukes remains a major public health problem in East Asia and Eastern Europe where $>40$ million people are infected. O. viverrini is endemic in Thailand, Lao PDR, Vietnam and Cambodia. Humans acquire the infection by eating raw or undercooked fish infected with the metacercaria (MC) stage of the parasite (reviewed in [1]). Upon ingestion, metacercariae excyst in the duodenum and juvenile flukes migrate into the biliary tree. In the bile ducts, the parasites mature over six weeks into adult flukes which graze on biliary epithelia. Parasite eggs are shed in the fecal stream and the eggs are ingested by freshwater snails. The parasite undergoes transformations within the snail host, culminating in the release of cercariae which seek out and penetrate the skin of a freshwater fish. The infection in humans causes hepatobiliary abnormalities, including cholangitis, obstructive jaundice,

\footnotetext{
This is an open-access article distributed under the terms of the Creative Commons Attribution-NonCommercial-No Derivative Works License, which permits non-commercial use, distribution, and reproduction in any medium, provided the original author and source are credited.

* Corresponding author. Tel.: + 351 220402567; fax: + 351220402563.

E-mail address: nuno.vale@fc.up.pt (N. Vale).
}

hepatomegaly, periductal fibrosis, cholecystitis and cholelithiasis [1]. Much more problematically, both experimental evidence and epidemiological evidence strongly implicate liver fluke infection in the etiology of one of the major liver cancer subtypes - cholangiocarcinoma (CCA), bile duct cancer [2].

Opisthorchiasis is associated with elevation of bile acids, including deoxycholic acid (3, Supplementary Table 1 ), which are potent tumor promoters in cholangiocarcinogenesis. Bile acids (Supplementary Table 1) are synthesized in the liver from cholesterol, and the majorities are conjugated with either glycine or taurine [3-7]. Inflammationrelated carcinogenesis has also been associated to oxidative and nitrative DNA damage as 8-oxo-7,8-hydro-2'-deoxiguanine (8-oxodG) and 8-nitroguanine (8-NG) [4]. Akaike et al. [5] demonstrated the close association of 8-nitroguanosine with $\mathrm{NO}$ production in mice with viral pneumonia. Increased levels of nitrate and nitrite, which reflect endogenous generation of $\mathrm{NO}$, have also been detected during 0 . viverrini infection in humans [6] and animals [7]. In view of the above, a study on nucleic acid damage by reactive nitrogen and oxygen species may contribute to clarification of mechanisms of carcinogenesis triggered by 0 . viverrini infection.

Oxysterols, which are oxidation products of cholesterol not generated by enzymatic or enzymatic (P450) reactions, have been shown to be mutagenic, genotoxic, and to possess pro-oxidative and pro-inflammation 
properties that can contribute to carcinogenesis. Binding domains in human gene studies have demonstrated an association between different types of oxysterols and the development and progression of cancer of the colon, lung, breast and bile ducts. Given our experience in LC-MS/MS studies towards drug development $[15,16,19-22]$ and understanding of the molecular basis of disease $[8,9]$, we set out to explore the prospective presence of novel steroids in 0 . viverrini, in line with recent findings on steroid metabolites reported by others and us $[8,10]$.

\section{Materials and methods}

\subsection{Extracts of O. viverrini liver flukes}

Metacercariae of 0 . viverrini were obtained from naturally infected cyprinoid fish in Khon Kaen province, Thailand. The fish were digested by pepsin- $\mathrm{HCl}$ method. After several washes with normal saline, metacercariae were collected and identified under a dissecting microscope. Metacercariae were used to infect hamsters (Mesocricetus auratus), which were maintained at the rodent facility of the Faculty of Medicine at Khon Kaen University. Protocols approved by the Khon Kaen University Animal Ethics Committee were used for this investigation (Animal Ethic No. AEKKU 05/2550). At about three months after infection, hamsters were euthanized and necropsied, and adult 0 . viverrini flukes recovered from their bile ducts. The worms were washed extensively in phosphate buffered saline (PBS, pH 7.4) containing antibiotics (100 $\mu \mathrm{g} / \mathrm{mL}$ streptomycin and $100 \mathrm{U} / \mathrm{mL}$ penicillin $\mathrm{G}$ ) and cultured overnight in serum free RPMI-1640 medium containing $1 \%$ glucose, and protease inhibitors (0.1 mM phenylmethanesulfonyl fluoride, $2 \mu \mathrm{M}$ E-64 and $10 \mu \mathrm{M}$ leupeptin) at $37{ }^{\circ} \mathrm{C}$ and $5 \% \mathrm{CO}_{2}$ [11]. Then, the worms were washed thoroughly with PBS. Soluble extracts of these worms were prepared by sonication $(5 \times 5 \mathrm{~s}$ bursts, output cycle 4 , Misonix Sonicator 3000 , Newtown, CT 06470, USA) in PBS supplemented with protease inhibitors $(500 \mu \mathrm{M}$ AEBSF, $\mathrm{HCl}, 150 \mathrm{nM}$ aprotinin, $1 \mu \mathrm{M}$ E-64, and $1 \mu \mathrm{M}$ leupeptin hemisulfate) (EMD Millipore, Calbiochem, Billerica, MA, USA), followed by 30 -min centrifugation at $10000 \mathrm{rpm}$ and $4^{\circ} \mathrm{C}$. Supernatants were collected and protein concentration was determined using the bicinchoninic acid assay (BCA kit, Pierce, Rockford, IL, USA). These soluble extracts of $O$. viverrini worms were stored in small aliquots at $-80{ }^{\circ} \mathrm{C}$ until needed.
Methanol was added to the soluble extract of 0 . viverrini worms (with lipidic fraction) up to $20 \%$ (volume/volume); $25 \mu \mathrm{L}$ of this solution was injected into the LC-MS/MS instrument for analysis. Methanol displayed excellent chromatographic performance (separation and sensitivity) with short gradient times possible for chromatographic separation with this solvent.

\section{2. $L C-M S / M S$ analysis}

High-performance liquid chromatography (HPLC) coupled with mass spectrometry (LC-MS) was employed to investigate relevant molecular species in the soluble extracts from adult 0 . viverrini worms. The HPLC module was constituted with a thermostatted automated sample injector and a thermostatted column compartment; the column was a $125 \times 4.6 \mathrm{~cm}$ Merck Purospher STAR RP-18e (3 $\mu \mathrm{m})$, equipped with a Merck Lichrocart pre-column (Merck, Germany) and the mobile phase consisted of $1 \%$ acetic acid in water $(A) /$ acetonitrile (B) mixtures; elution was carried out at a flow rate of $0.3 \mathrm{~mL} / \mathrm{min}$, with the following program: $0-5 \mathrm{~min}, 100 \% \mathrm{~A}$; 5-10 min, linear gradient from $100 \%$ to $80 \% \mathrm{~A} ; 10-15 \mathrm{~min}, 80 \% \mathrm{~A} ; 15-50 \mathrm{~min}$, linear gradient from 80 to $40 \%$ A; 50-65 min, 40\% A; 65-75 min, linear gradient from $40 \%$ A to $100 \%$ $\mathrm{B}$; detection was achieved on a diode array detector (DAD) at $280 \mathrm{~nm}$. MS analysis relied on a Finnigan Surveyor LCQ XP MAX quadrupole ion trap mass spectrometer (Thermo Electron Corporation, San Jose, CA, USA) utilizing electrospray ionization. Mass spectra were acquired in the negative mode (species mainly detected as $[\mathrm{M}-\mathrm{H}]^{-}$ions or $\mathrm{M}^{-}$).

\section{Results}

The introduction and refinement of LC-MS/MS methods have made the analysis of conjugated bile acids possible with high sensitivity, minimal sample size requirements and simplified sample preparation procedures [29]. In view of this, we developed an LC-MS/MS approach to search for new steroid-based molecules whose presence in extracts of 0 . viverrini worms we had postulated on the basis of bile acids and the metabolism of estrogens [8,10]. Fig. 1 depicts the full chromatogram (MS detection) obtained for the worm extracts, and relevant data from MS analysis are presented in Table 1, along with the structures postulated to be associated with each of the main species detected here.



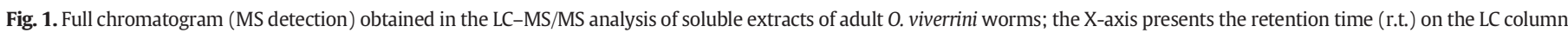
and the Y-axis shows the relative abundance of each component. 
Table 1

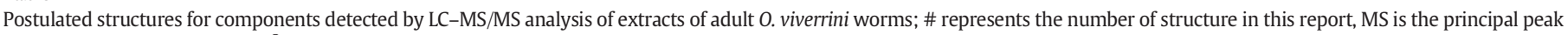
observed in mass spectrum, $\mathrm{MS}^{2}$ is the first fragmentation of principal peak observed in MS, and r.t. is the retention time.

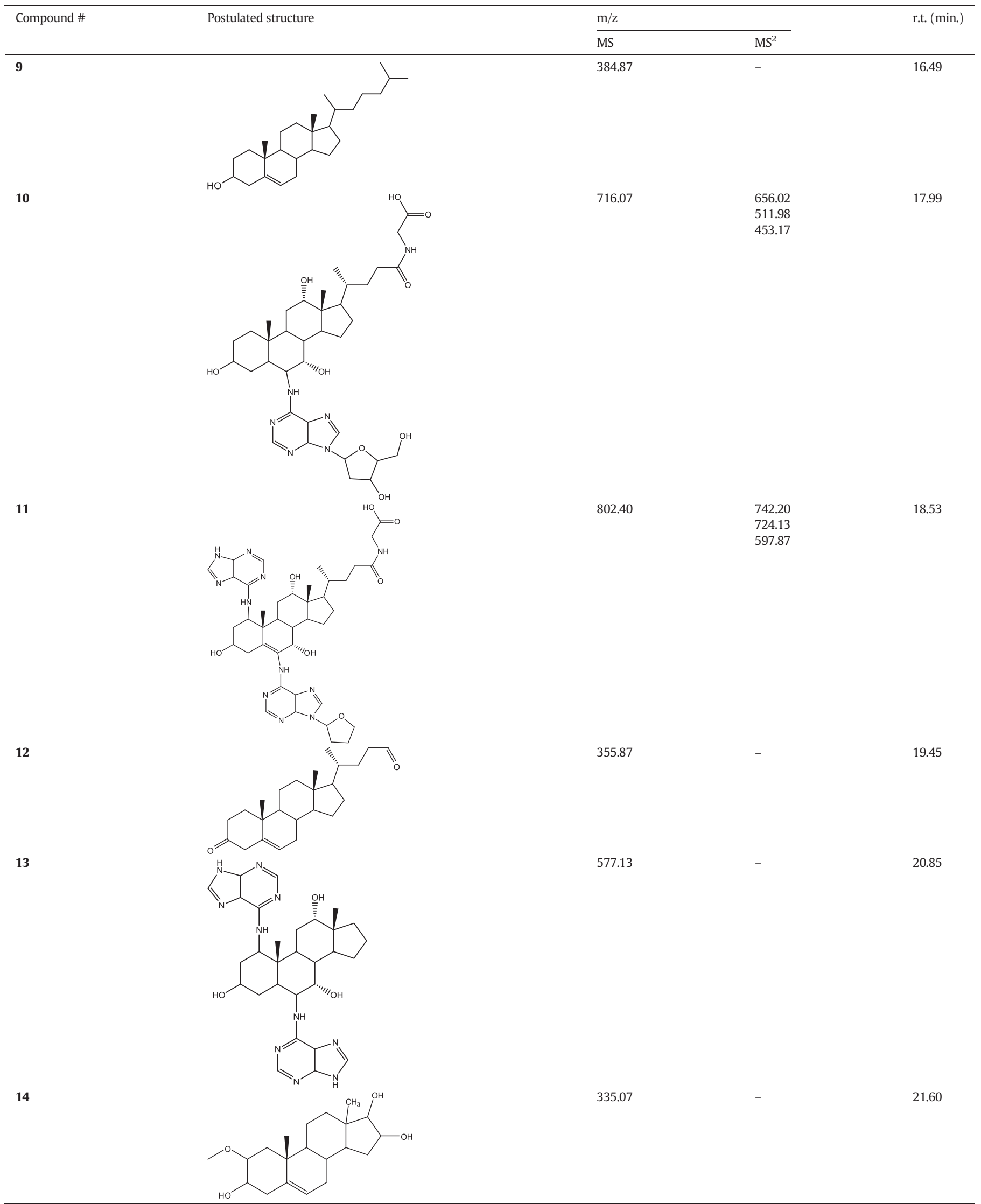


Table 1 (continued)

\begin{tabular}{|c|c|c|c|c|}
\hline \multirow[t]{2}{*}{ Compound \# } & \multirow[t]{2}{*}{ Postulated structure } & \multicolumn{2}{|l|}{$\mathrm{m} / \mathrm{z}$} & \multirow[t]{2}{*}{ r.t. (min.) } \\
\hline & & MS & $\mathrm{MS}^{2}$ & \\
\hline 15 & & 357.13 & - & 30.45 \\
\hline 16 & & 438.93 & - & 38.84 \\
\hline 17 & & 667.20 & $\begin{array}{l}515.07 \\
497.00 \\
541.27\end{array}$ & 39.20 \\
\hline 18 & & 455.13 & - & 41.90 \\
\hline 19 & & 329.47 & - & 43.12 \\
\hline 20 & & 495.13 & $\begin{array}{l}407.20 \\
450.72 \\
340.80\end{array}$ & 45.20 \\
\hline 21 & & 280.87 & - & 48.47 \\
\hline
\end{tabular}

As shown on Table 1, most of the proposed structures present C-17 ramification (i.e. discrete and variable chains linked to carbon 17 of the steroid ring), in similar fashion to bile acids and their conjugated salts. Also, MS data are compatible with the presence of derivatives of catechol-estrogens and other components hydroxylated at the steroid ring, including at both C-2 and C-3 and respective oxidized 2,3-quinone.

Some oxidative metabolites of estrogens can react with DNA and the mutations resulting from these adducts can lead to cell transformation. 
Table 1 (continued)

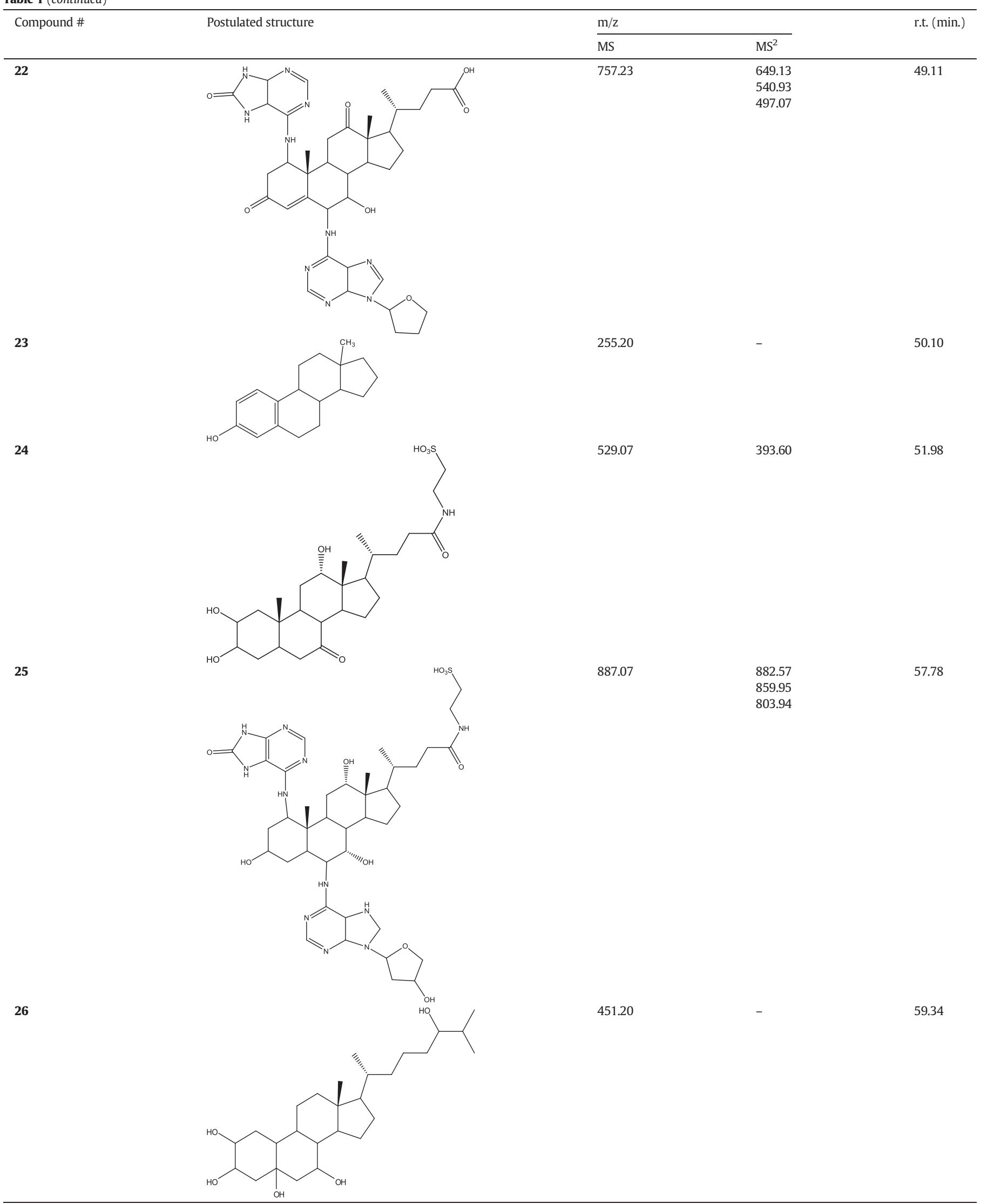





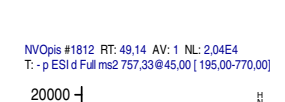
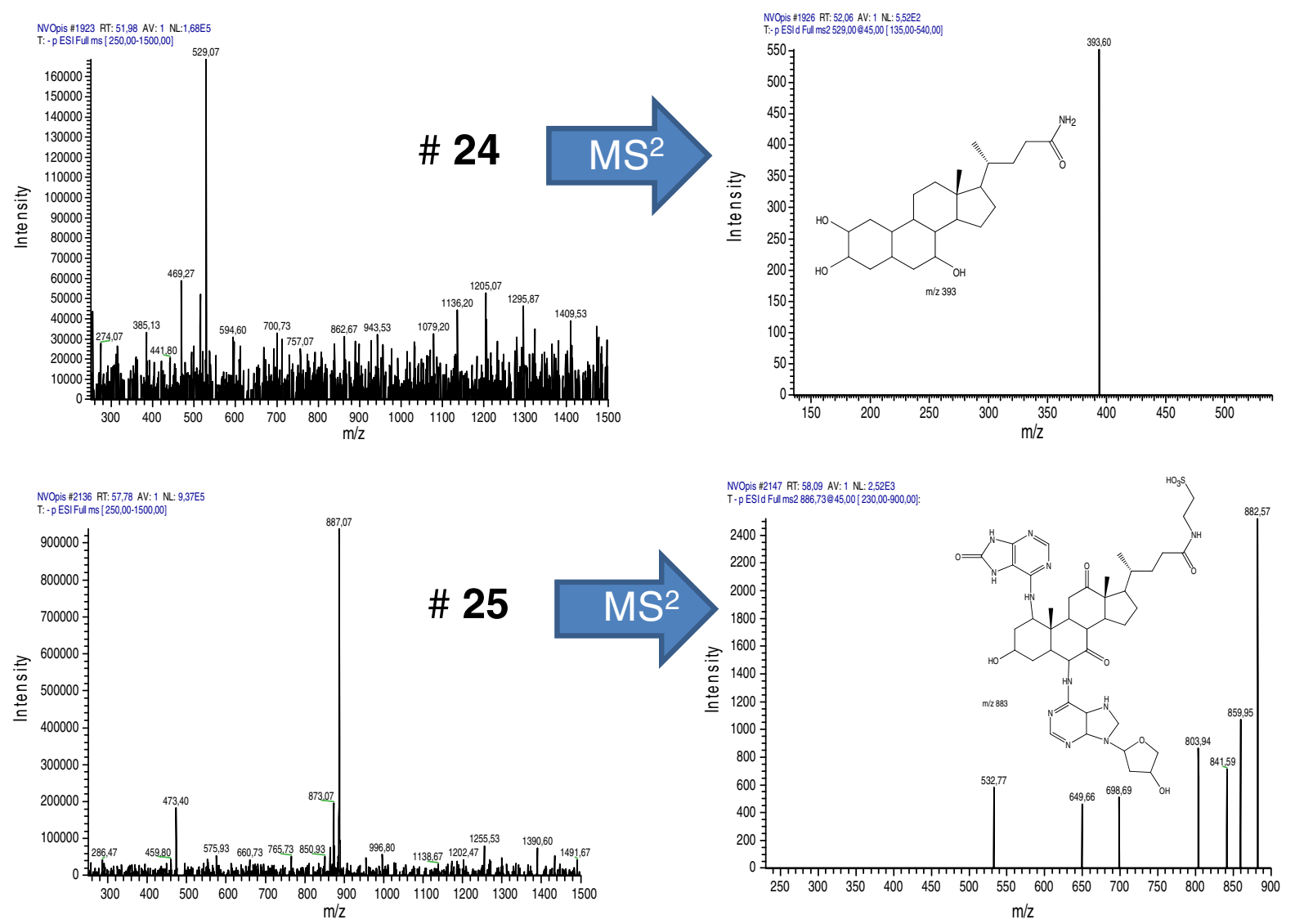

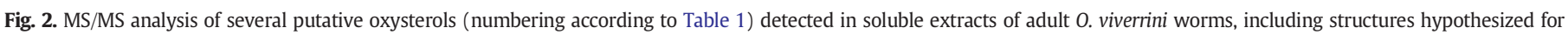
fragments. 
Bile acids constitute a large family of steroids carrying a carboxyl group in the side chain, e.g. 10, 11, 11 and 22. Bile alcohols have similar products in bile acid biosynthesis or as end products, and we can see these compounds in 18, 19 and 26, but conjugated at different positions. Free bile acids are reconjugated in some species like aldehydes (12 and $\mathbf{1 5}$ ) or as sulfates $(\mathbf{2 0}, \mathbf{2 4}$ and $\mathbf{2 5})$. The effects of these individual species can be anticipated to be structure-dependent, and metabolic conversions will result in a complex mixture of biologically active and inactive forms.

\section{Discussion}

Chronic infection by the liver fluke 0 . viverrini is associated with carcinogenesis of CCA. ROS/RNS generated during inflammatory response to 0 . viverrini possess highly oxidative potential [12]. These oxidative species could react with biomolecules in inflammatory area which include $O$. viverrini residing in the bile duct. Cholesterol is one of the targets of the free radicals. Here, we identified oxidized derivatives, oxysterols, from the liver fluke 0 . viverrini, which might - at least in part - be generated from non-enzymatic reaction by the oxidative free radicals. It is also feasible that these novel oxysterols are produced enzymatically by the liver fluke, and in this regard, the prospective occurrence of cholesterol metabolic enzymes in fluke tissues deserves to be investigated. On the other hand, 0 . viverrini worms reside within the biliary tree, bathed in bile, and hence some of these oxysterol like species may be of host origin. Regardless of the provenance of these metabolites, the parasite might take up and/or utilize these oxysterols for its own needs or benefit.

Since oxysterols can traverse cell membranes more quickly than cholesterol, oxysterols of 0 . viverrini origin might enter biliary epithelia and contribute to 0 . viverrini-associated cholangiocarcinoma. Moreover, oxysterols may contribute at several stages of carcinogenesis; 1 ) tumor initiation by enhancing the production of ROS/RNS and 2) tumor promotion through upregulated expression COX-2 leading to the alteration of cellular phenotypes. Also, oxysterols may support cancer progression through the induction of migration and/or may exert their effect by binding to specific proteins and activating signaling cascades. Oxysterols have been identified in livers of hamsters infected with 0 . viverrini that develop cholangiocarcinoma; among others, both Triol and 3K4 occur at elevated levels in the livers of hamsters with 0 . viverrini-induced cholangiocarcinoma. In concert with the novel liver fluke oxysterols reported here they might contribute to cholangiocarcinoma through formation of DNA adducts and dysregulation of apoptosis and other homeostatic pathways $[14,15]$.

Most of the bile acids excreted to the bile duct are reabsorbed in the intestine and return to the liver via the portal vein. Reabsorbed bile acids act as a negative regulator of the rate-limiting enzyme for cholesterol catabolism termed cholesterol 7 alpha-hydroxylase (CYP7A1) in the liver, which in turn slows down bile acid production [13]. The oxysterols in 0 . viverrini adult worms are released into the bile duct, some of them are structurally similar to bile acids, and might act the same way as bile acids to down regulate the bile acid metabolism. In turn, this might contribute to digestive dysfunction that is not uncommon during opisthorchiasis [16], and hence further investigation of this issue can be expected to be informative. Nonetheless, whereas we anticipate that similar physiological phenomena take place in infected humans, the present study investigated worms from experimentally infected hamsters.

A water soluble lysate of $O$. viverrini liver flukes was investigated here because we wanted to investigate soluble forms of oxysterol(s). This would likely reflect physiological forms released/exported/secreted from the liver fluke in vivo. Since we wondered whether oxysterols might be involved in carcinogenesis of liver fluke induced bile duct cancer, it seems likely that soluble forms would be transferred from the worm to surrounding tissues. One possible mechanism for transferring of these oxysterols is binding to carrier proteins, including fatty acid binding proteins in tegumental membrane and excretory-secretory products of 0 . viverrini [17]. Fatty acid binding proteins are members of lipid- binding protein family that serve as carrier proteins for hydrophobic ligand trafficking [18]. The mechanism and role of these proteins in 0 . viverrini also are being investigated in our laboratories.

MS analysis alone cannot provide a conclusive structural assignment. Nonetheless, we have used caution in interpreting the new LCMS data in light of what has been reported/is known about metabolism of bile acids and estradiol [8,19-21]. Furthermore, our structural interpretation of MS data for the postulated novel oxysterols is further supported by MS fragmentation analysis, presented in Fig. 2.

We have postulated structures based only on the mass of the $[\mathrm{M}-\mathrm{H}]^{-}$ ion associated to each component detected by the LC-MS/MS analysis of 0 . viverrini extracts. However, in view of all the above, and based on the literature available about bile acids and the metabolism of estrogenrelated biomolecules $[19,20]$, the structures presented in Table 1 are likely to be valid. Some of these structures represent the estrogen core with minimal or only a few changes, e.g. 9, 12-15, 23 and $\mathbf{2 4}$. The majority of these exhibit C-17 ramifications, similar to structures of bile acids and their conjugated salts e.g. 10, 11, 17-20, 22, 25 and $\mathbf{2 6}$ (Table 1). In addition, structure $\mathbf{1 8}$ is compatible with a pattern of steroid-ring hydroxylation on both C-2 and C-3 and further oxidation into an estradiol-2,3quinone. Overall, the proposed structures suggest that carcinogenesisrelated steroids may be present in significant amounts in the adult 0 . viverrini liver fluke. It also should be noted that a relation between putative oxysterol or bile acid metabolites from 0 . viverrini and bile duct cancer has been previously hypothesized, in pioneering studies by Thai investigators during 1980s [22].

The chemical structures of oxysterols vary depending upon the number and position of oxygenated functional group, and include keto-, hydroxyperoxy, and epoxy forms. Enzymatic pathways that result in production of oxysterols mainly involve cytochrome P450 family enzymes, but certain oxysterols are produced by non-enzymatic oxidation (or auto-oxidation), a process that involves reactive oxygen and nitrogen species [23]. Oxysterols can act as intermediates in cholesterol catabolism, especially in synthesis of bile acids [24,25]. Bile acids, in turn, are acidic molecules with pKa values of $\sim 1.5$ for taurine-conjugated, $\sim 4.5$ for glycine-conjugated, and $\sim 6.0$ for unconjugated bile acids [26]. Thus, detection of bile acids and respective conjugates in ionized/non-ionized forms will depend on the $\mathrm{pH}$ of the mobile phase $[27,28]$. In our case, where a slightly acidic mobile phase was employed, taurine conjugates would be mainly deprotonated, and glycine and unconjugated bile acids would occur predominantly in the non-ionized form.

Cytochrome P450 enzymes play a critical role in both estrogen formation and its subsequent oxidative metabolism. The biosynthesis of estrogen hormones from androgens is mediated by the action of aromatase, a cytochrome P450 specific enzyme. Subsequent to their synthesis, estrogens undergo extensive metabolism [10]. The oxygenated metabolites of estrogens represent structures with newly generated hydroxyl and keto functions at specific sites in the steroid nucleus, which are analogous to other steroid categories that undergo oxidative metabolism, as androgens, vitamin D and bile acids [29]. The estrogens also share with other steroids similar modes of conjugative metabolism involving the formation of sulfates and glucoronides. Recently, we reported novel estrogenic conjugates with guanine [8], and although steroid and/or bile acid metabolites apparently have not been reported before for 0 . viverrini, formations of 8-oxodG and 8-NG have been described in the course of 0 . viverrini infection [4].

To conclude, advances in the methods of LC-MS/MS have facilitated the analysis of conjugated bile acids with high sensitivity, requiring only minimal quantities of the samples, and simplified sample preparation procedures [19]. As such, LC-MS/MS was utilized in this study, which enabled us to putatively identify novel oxysterols in adult 0 . viverrini liver flukes. Whereas the role of these new oxysterols of 0 . viverrini in bile duct cancer remains to be examined, this topic clearly is worthy of deeper investigation. Future studies will aim at isolation or chemical synthesis of some of these oxysterols and downstream investigation of interactions of the fluke oxysterols with informative cells such as 
cholangiocytes [14] and with oxysterol binding proteins (OSP). Given that other metabolites of $O$. viverrini also are predicted to play a role in tumorigenesis of 0 . viverrini induced bile duct cancer, including liver fluke granulin [30], it will be informative also to compare and contrast action of liver fluke granulin and the fluke oxysterols in these analyses. OSP are potential biomarkers of liver fluke induced liver cancer [15]. As ligands of OSP, 0 . viverrini-derived oxysterols and related metabolites may become useful biomarkers for 0 . viverrini infection and associated diseases [31].

Supplementary data to this article can be found online at http://dx. doi.org/10.1016/j.parint.2013.08.001.

\section{Conflict of interest}

There is no conflict of interest.

\section{Acknowledgments}

PG and NV thank Fundação para a Ciência e Tecnologia (FCT, Portugal) and FEDER (European Union) for funding through project grants CONCREEQ/275/QUI and PEst-C/QUI/UI0081/2011. NV also thanks FCT for Post-Doc grant SFRH/BPD/48345/2008. JMCC thanks also FCT for PestOE/AGR/UI0211/2011 and Strategic Project UI211-2011-2013. PJB received support from awards R01CA155297 and R01CA164719 from the National Cancer Institute. The content is solely the responsibility of the authors and does not necessarily represent the official views of the $\mathrm{NCI}$ or $\mathrm{NIH}$.

\section{References}

[1] Sithithaworn P, Andrews RH, Van De N, Wongsaroj T, Sinuon M, Odermatt P, et al. The current status of opisthorchiasis and clonorchiasis in the Mekong Basin. Parasitol Int 2012;61:10-6.

[2] Sripa B, Pairojkul C. Cholangiocarcinoma: lessons from Thailand. Curr Opin Gastroenterol 2008;24:349-56.

[3] Sirica AE. Cholangiocarcinoma: molecular targeting strategies for chemoprevention and therapy. Hepatology 2005;41:5-15.

[4] Yongvanit P, Pinlaor S, Bartsch H. Oxidative and nitrative DNA damage: keys event in opisthorchiasis-induced carcinogenesis. Parasitol Int 2012;61:130-5.

[5] Akaike T, Okamoto S, Sawa T, Yoshitake J, Tamura F, Ichimori K, et al. 8-Nitroguanosine formation in viral pneumonia and its implication for pathogenesis. Proc Natl Acad Sci U S A 2003;100:685-90.

[6] Haswell-Elkins MR, Satarug S, Tsuda M, Mairing E, Esumi H, Sithithaworn P, et al. Liver fluke infection and cholangiocarcinoma: model of endogenous nitric oxide and extragastric nitrosation in human carcinogenesis. Mutat Res 1994;305:241-52.

[7] Ohshima H, Bandaletova TY, Brouet I, Bartsch H, Kirby K, Ogunbiyi F, et al. Increased nitrosamine and nitrate biosynthesis mediated by nitric oxide synthase induced in hamster infected with liver fluke (Opisthorchis viverrini). Carcinogenesis 1994;15: 271-5.

[8] Botelho MC, Soares R, Vale N, Ribeiro R, Camilo V, Almeida R, et al. Schistosoma haematobium: identification of new estrogenic molecules with estradiol antagonistic activity and ability to inactive estrogen receptor in mammalian cells. Exp Parasitol 2010;126:526-35
[9] Vale N, Fernandes I, Moreira R, Mateus N, Gomes P. Comparative analysis of in vitro rat liver metabolism of the antimalarial primaquine and a derived imidazoquine. Drug Metab Lett 2012;11:15-25.

[10] Martucci CP, Fishman C. P450 enzymes of estrogen metabolism. Pharmacol Ther 1993;57:237-57.

[11] Suttiprapa S, Loukas A, Laha T, Pongkham S, Kaewkes S, Gaze S, et al. Characterization of the antioxidant enzyme, thioredoxin peroxidase, from the carcinogenic liver fluke, Opisthorchis viverrini. Mol Biochem Parasitol 2008;120:116-22.

[12] Kawanishi S, Hiraku Y, Pinlaor S, Ma N. Oxidative and nitrative DNA damage in animals and patients with inflammatory disease in relation to inflammation-related carcinogenesis. Biol Chem 2006;387:365-72.

[13] Björkhem I. Do oxysterols control cholesterol homeostasis? J Clin Invest 2002;110: 725-30.

[14] Jusakul A, Loilome W, Namwat N, Haigh WG, Kuver R, Dechakhamphu S, et al. Liver fluke-induced hepatic oxysterols stimulate DNA damage and apoptosis in cultured human cholangiocytes. Mutat Res 2012;731:48-57.

[15] Loilome W, Wechagama P, Namwat N, Jusakul A, Sripa B, Miwa M, et al. Expression of oxysterol binding protein isoforms in opisthorchiasis-associated cholangiocarcinoma: a potential molecular marker for tumor metastasis. Parasitol Int 2012;61:136-9.

[16] Mairiang E, Mairiang P. Clinical manifestation of opisthorchiasis and treatment. Acta Trop 2003;88:221-7.

[17] Mulvenna J, Sripa B, Brindley PJ, Gorman J, Jones MK, Colgrave ML, et al. The secreted and surface proteomes of the adult stage of the carcinogenic human liver fluke Opisthorchis viverrini. Proteomics 2010;10:1063-78.

[18] Smathers RL, Petersen DR. The human fatty acid-binding protein family: evolutionary divergences and functions. Hum Genomics 2011;5:170-91.

[19] Gouveia MJ, Botelho M, Brindley PJ, Costa JMCC, Gomes P, Vale N. Mass spectrometry techniques in the survey of steroid metabolites as potential disease biomarkers: a review. Metabolism 2013 [in press].

[20] Cavalieri EL, Rogan EG. Unbalanced metabolism of endogenous estrogens in the etiology and prevention of human cancer. J Steroid Biochem Mol Biol 2011;125:169-80.

[21] Lövgren-Sandlom A, Heverin M, Larsson H, Lundström E, Wahren J, Diczfalusy U, et al. Novel LC-MS/MS method of assay of 7a-hydroxy-4-cholesten-3-one in human plasma. Evidence for a significant extrahepatic metabolism. J Chromatogr A 2007;856:15-9.

[22] Migasena S, Egoramaiphol S, Tungtrongchitr R, Migasena P. Study on serum bile acids in opisthorchiasis in Thailand. J Med Assoc Thai 1983;66:464-9.

[23] Brown AJ, Jessup W. Oxysterols: sources, cellular storage and metabolism, and new insights into their roles in cholesterol homeostasis. Mol Aspects Med 2009;30: 111-22.

[24] Diczfalusy U, Lund E, Lutjohann D, Bjorkhem I. Novel pathways for elimination of cholesterol by extrahepatic formation of side-chain oxidized oxysterols. Scand J Clin Lab Invest 1996;226:9-17.

[25] Meaney S, Bodin K, Diczfalusy U, Bjorkhem I. On the rate of translocation in vitro and kinetics in vivo of the major oxysterols in human circulation: critical importance of the position of the oxygen function. J Lipid Res 2002;43:2130-5.

[26] Scalia S. Bile acid separation. J Chromatogr B Biomed Sci Appl 1995;671:299-317.

[27] Bobeldijk L, Hekman J, de Vries-van der Weij J, Coulier L, Ramaker R, Kleemann R, et al. Quantitative profiling of bile acids in biolfluids and tissues based on accurate mass high resolution LC-FT-MS: compound class targeting in metabolomics workflow. J Chromatogr B Analyt Technol Biomed Life Sci 2008;871:306-13.

[28] Burkard L, von Eckardstein A, Rentsch KM. Differentiated quantification of human bile acids in serum by high performance liquid chromatography-tandem mass spectrometry. J Chromatogr B Analyt Technol Biomed Life Sci 2005;826:147-59.

[29] Young ND, Campbell BE, Hall RS, Jex AR, Cantacessi C, Laha T, et al. Unlocking the transcriptomes of the carcinogens Clonorchis sinensis and Opisthorchis viverrini. PLoS Negl Trop Dis 2010;4:e719.

[30] Sripa B, Brindley PJ, Mulvenna J, Laha T, Smout MJ, Mairiang E, et al. The tumorigenic liver fluke Opisthorchis viverrini - multiple pathways to cancer. Trends Parasitol 2012;28:395-407.

[31] Porter FD, Scherrer DE, Lanier MH, Langmade SJ, Molugu V, Gale SE, et al. Cholestero oxidation products are sensitive and specific blood-based biomarkers for NiemannPick C1 disease. Sci Transl Med 2010;2:56ra81. 